\title{
Response of Uranus' auroras to solar wind compressions at equinox
}

\author{
S. W. H. Cowley ${ }^{1}$ \\ Received 8 February 2013; revised 8 April 2013; accepted 7 May 2013; published 18 June 2013.
}

[1] We consider open flux production and magnetic tail formation for the unique physical circumstances at Uranus, where the planet's spin axis lies close to the orbit plane, while the magnetic dipole has a large $\sim 60^{\circ}$ inclination to the spin axis. Under these circumstances, open flux production and transport into the nightside can in principle occur continuously for near-solstice conditions, leading to the formation of a well-developed bipolar rotating magnetic tail, as observed during the Voyager 2 flyby in 1986. However, it is argued that tail formation will be significantly inhibited near to equinox, as open tubes are wound over the dayside magnetopause by planetary rotation, thus reducing further open flux production, and are compressed together north or south of the planet promoting "tail" reconnection and open flux closure. If so, this may account for the weak auroral responses to predicted solar wind compressions of Uranus' magnetosphere reported recently from Hubble Space Telescope observations under near-equinoctial conditions. Major auroral responses are observed following strong compressions at Earth and Saturn resulting from rapid and substantial closure of preexisting open flux in the tail. The lack of such a response at Uranus near equinox may thus reflect the lack of a well-developed tail under these conditions.

Citation: Cowley, S. W. H. (2013), Response of Uranus' auroras to solar wind compressions at equinox, J. Geophys. Res. Space Physics, 118, 2897-2902, doi:10.1002/jgra.50323.

\section{Introduction}

[2] The polar auroras of the magnetized planets are known to respond significantly to strong compressions of the magnetosphere resulting from rapid increases in the dynamic pressure of the solar wind. In the case of the Earth, auroral enhancements by factors of $\sim 2-4$ are observed over a broad range of latitudes and local times, sometimes propagating rapidly from noon to midnight, as trapped particles in the magnetosphere are energized by the compression and scattered into the loss cone [e.g., Zhou and Tsurutani, 1999; Tsurutani et al., 2001; Boudouridis et al., 2003]. More importantly, however, the emission in the auroral oval is often observed to intensify and expand significantly poleward, particularly on the nightside, with strongest effects being observed following intervals of southward interplanetary magnetic field (IMF) when the preexisting open flux in the tail is enhanced [e.g., Zesta et al., 2000; Boudouridis et al., 2003, 2004, 2005]. The total auroral power emitted can increase from pre-compression values by more than an order of magnitude during such events. Poleward expansion of the auroras is indicative of rapid reduction in the open flux in the system resulting from strong enhancements of the reconnection rate in the tail, from typical values of a few tens of $\mathrm{kV}$ up to $\sim 100 \mathrm{kV}$ or more [Milan et al., 2004; Hubert

\footnotetext{
${ }^{1}$ Department of Physics and Astronomy, University of Leicester, Leicester, UK.

Corresponding author: S. W. H. Cowley, Department of Physics and Astronomy, University of Leicester, Leicester LEI 7R, UK. (swhc1@ion.le.ac.uk)

(C2013. American Geophysical Union. All Rights Reserved.

2169-9380/13/10.1002/jgra.50323
}

et al., 2006; Boudouridis et al., 2008]. It may be relevant to note in this regard that for a given amount of open flux in the tail, the current density in the plasma sheet separating the lobes should vary inversely as the cube of the linear dimension of the system, and thus roughly in proportion to the square root of the solar wind dynamic pressure. Some current-driven instability may therefore be implicated.

[3] Similarly in the case of Saturn, magnetospheric compressions are found to produce brightening and strong poleward contraction of the auroras on the dawnside of the auroral oval, subsequently evolving into a bright spiral structure [Prangé et al., 2004; Clarke et al., 2005, 2009; Bunce et al., 2006]. Total ultraviolet (UV) auroral powers are found to increase in this case by factors of $\sim 3-5$, from quiet time values of $\sim 5-10 \mathrm{GW}$ up to $25-30 \mathrm{GW}$. Cowley et al. [2005] suggested that this effect also results from closure of a significant fraction of the open flux in the tail, followed by sweeping of the newly closed flux tubes around the planet via dawn due to magnetosphere-ionosphere coupling to the rapid planetary rotation. Open flux is expected to accumulate slowly in Saturn's tail over intervals of many days due to the low dayside reconnection rates expected in the outer solar system, with typical voltages of only a few tens of $\mathrm{kV}$ [Jackman et al., 2004; Badman and Cowley, 2007]. This accumulation may then often be terminated by bursts of tail reconnection excited by shock-associated solar wind compressions of the magnetosphere that occur at the planet on time scales of some fraction of the solar rotation period [Jackman et al., 2004; Badman et al., 2005].

[4] In the case of Jupiter, enhancements in UV auroral power by factors of $\sim 2-3$, from $\sim 300$ to $\sim 700 \mathrm{GW}$, have also been reported to occur in response to solar wind compressions [Baron et al., 1996; Pryor et al., 2005; Clarke et al., 
2009]. However, these increases are found to be confined principally to the main oval and lower latitude auroras mapping to the middle and inner magnetosphere [Clarke et al., 2009]. They may therefore have more in common with the lower latitude brightenings observed at Earth, rather than with the effects attributed to rapid open flux closure at both Earth and Saturn. However, one instance has been reported which may be related to the latter effects, in which highlatitude patchy "swirl" auroras were observed to expand to cover much of Jupiter's polar region during a compression event [Nichols et al., 2007].

[5] In a recent Hubble Space Telescope (HST) campaign undertaken in 2011, Lamy et al. [2012] attempted to detect UV auroral emissions from Uranus, looking particularly for responses to strong enhancements in the solar wind dynamic pressure such as those observed at Earth and Saturn. For this purpose, a computational model was employed to map solar wind properties from the inner heliosphere outward to Uranus. Some auroral responses were indeed reported, but taking the form of weak spot-like patches of emission, unlike the bright extended auroras observed at Earth and Saturn under these circumstances. To observe strong responses of the latter kind, however, a well-developed magnetic tail is required to have been present prior to the compression, mapping to an extended open field region in the planetary ionosphere, as was indeed observed at Uranus during the Voyager 2 flyby in 1986. However, the Voyager 2 flyby occurred under conditions of northern summer solstice, while the above HST campaign took place near the following autumnal equinox. In this report, we thus consider tail formation at Uranus under equinoctial conditions and argue that due to the unique configuration of spin and magnetic axis orientations then occurring, it may be difficult to grow and sustain a significant open tail, thus potentially accounting for the findings of Lamy et al. [2012].

\section{The Uranus System}

\subsection{Voyager 2 Observations of Uranus' Auroras and Magnetic Field}

[6] Uranus' auroras were first unambiguously observed in measurements obtained by the UV spectrometer on the Voyager 2 spacecraft during its flyby of the planet in January 1986 [Broadfoot et al., 1986]. These took the form of weak $(\sim 1 \mathrm{kR})$ patchy emissions from atomic and molecular hydrogen in the vicinity of the two magnetic poles [Herbert and Sandel, 1994; Herbert, 2009]. Specifically, the emissions were found to span a $\sim 90^{\circ}$ nightside longitude band typically at $\sim 20^{\circ}-25^{\circ}$ magnetic latitude, mapping to the central quasi-dipolar magnetosphere at equatorial radial distances of $\sim 5-10 R_{U}$. $\left(R_{U}\right.$ is Uranus' equatorial radius equal to $25,559 \mathrm{~km}$.) A major difference between the magnetosphere of Uranus and those of Earth, Jupiter, and Saturn, however, results from the large $\sim 60^{\circ}$ inclination of the magnetic dipole axis to the spin axis at Uranus [Ness et al., 1986; Connerney et al., 1987], compared with the nearaxial orientations at the latter planets. The magnetic poles and surrounding auroral emissions at Uranus thus approach the spin equator of the planet rather than being located near the spin poles, though rather more nearly so for the northern magnetic pole in the northern hemisphere than for the southern magnetic pole in the southern hemisphere, due to the significant $\sim 0.3 R_{U}$ offset of the magnetic dipole along the spin axis into the southern hemisphere. (Here we employ the usual planetary definitions of "north" and "south" with respect to the angular velocity vector of the planet, opposite to the International Astronomical Union convention for Uranus.) Furthermore, the spin axis lies within $\sim 10^{\circ}$ of the orbital plane of the planet, rather than being more nearly perpendicular to it as for the other planets, and during the Voyager 2 flyby was pointed closely toward the Sun, corresponding to northern hemisphere solstice. In this case, the dipole axis was again directed near-perpendicular to the solar wind flow as a first approximation, similar to Earth, Jupiter, and Saturn, but rotated around the solar-pointing spin axis with the planetary rotation period. The latter period derived from Voyager 2 radio data was $\sim 17.24 \pm 0.01 \mathrm{~h}$ [Desch et al., 1986].

[7] The magnetospheric magnetic structure observed by Voyager 2 was thus not unlike that of Earth, Jupiter, and Saturn, but rotated around the spin axis at the above period [Ness et al., 1986; Connerney et al., 1987; Herbert, 2009]. On the dayside, the planetary field extended to a sub-solar magnetopause at $\sim 18 R_{U}$. On the nightside, a welldeveloped bipolar magnetic tail was observed having a radius of $\sim 40 R_{U}$, in which the field lines were observed to be wound into weak helices by the planetary-period rotation of the system [Behannon et al., 1987]. With a lobe field strength of $\sim 2 \mathrm{nT}$ in the near-planet region, each lobe contained $\sim 3 \times 10^{9} \mathrm{~Wb}$ of open flux, which, mapped to a typical surface magnetic polar field strength of $\sim 50,000 \mathrm{nT}$ [e.g., Connerney et al., 1987], corresponds to an open field region extending $\sim 10^{\circ}$ of magnetic co-latitude from each magnetic pole (compared to $\sim 20^{\circ}-25^{\circ}$ for the main auroral emissions as indicated above).

[8] If a sudden compression of the magnetosphere caused a rapid closure of a significant fraction of this open flux similar to that inferred at Earth and Saturn, leading to the production of hot plasma on newly closed tail field lines, the consequent precipitation would produce enhanced auroral features close to the magnetic poles with linear dimensions corresponding to the size of the open flux region, having a typical diameter $\sim 20 \%$ of that of the planet itself. (Here for simplicity, we ignore the factor of $\sim 3$ difference between the field strengths at the northern and southern poles due to the offset nature of the planetary dipole that would result in somewhat larger dimensions in the weaker field region in the north and somewhat smaller dimensions in the stronger field region in the south.) In addition, emissions may also be enhanced on closed flux tubes surrounding the previous open field region through energization and scattering of trapped hot plasma, as observed at Earth and also possibly at Jupiter.

[9] The properties of the patchy auroras observed by Voyager 2, generally located on nightside field lines mapping to the central magnetosphere as indicated above, appear not to follow these expectations for compression-enhanced emissions, even though solar wind data obtained by the spacecraft before entry and after exit show that the $\sim 2$ day magnetosphere pass took place shortly after the beginning of an $\sim 1$ week interval of compression events (data available through http://cdaweb.gsfc.nasa.gov/). Judging from related solar wind variations observed during adjacent solar rotations, these intervals involved a number of strong irregular $\sim 1-2$ day enhancements in the solar wind density with 
smaller changes in the velocity, the timing of which during the flyby is of course unknown once the spacecraft had entered Uranus' magnetosphere. However, substorm-like disturbances were not observed in the tail until $\sim 30 \mathrm{~h}$ after closest approach and $\sim 6 \mathrm{~h}$ before exit from the magnetosphere [Behannon et al., 1987; Mauk et al., 1987], such that it seems likely that the UV emissions observed during the close-encounter period had yet to be strongly affected by the compression events. This remains a somewhat open issue, of course. In the next subsection, we review the results of an attempt to observe compression-enhanced auroras during the recent intensive Uranus HST campaign outlined in section 1 .

\subsection{Uranus HST Campaign}

[10] In late 2011, a Uranus HST campaign was undertaken in which the observing visits were timed to straddle the anticipated arrivals of strong solar wind compressions at the planet. The timing of the compressions was estimated by tracking coronal mass ejections observed in the inner heliosphere by the STEREO spacecraft past Earth and Jupiter to Uranus with the aid of a MHD solar wind model (Saturn not being suitably located during the interval) [Lamy et al., 2012]. Two previous attempts to detect UV auroral emissions from Uranus using the HST had been made, in 1998 and 2005, without published result. The general difficulty of detecting UV auroras at Uranus is unsurprising given the few $\mathrm{kR}$ emissions observed by Voyager 2, two orders of magnitude less bright than the emissions from Jupiter and an order less than the brighter emissions from Saturn, combined with distances from the Sun of $\sim 20$ AU at Uranus, compared with $\sim 5$ and $\sim 10 \mathrm{AU}$ at Jupiter and Saturn. Correspondingly, the total UV auroral power from Uranus observed by Voyager 2 was $\sim 5 \mathrm{GW}$ [Herbert and Sandel, 1994], compared with values in the range $\sim 5-30 \mathrm{GW}$ at Saturn and $~ 300-700 \mathrm{GW}$ at Jupiter, as indicated in section 1. The hypothesized enhanced emissions resulting from solar wind compressions thus potentially represent the best conditions for detecting Uranus' auroras from Earth orbit.

[11] In the 2011 HST campaign, suitable solar wind disturbances were successfully tracked via Earth to Jupiter's distance using Jovian radio emissions and were predicted to have produced significant dynamic pressure variations at Uranus. These were successfully encompassed before and after by HST visits, with potential auroral responses being observed in two cases [Lamy et al., 2012]. The "response" auroras consisted in each case of an $\sim 1-2 \mathrm{kR}$ spot of emission, similar in brightness to the Voyager 2 emissions, located at a planetographic latitude similar to the Voyager 2 emissions associated with the northern magnetic pole (the spin phase having long been lost due to the uncertainty in the spin period). Not only do such responses not conform to expectations for compression-enhanced auroras as discussed above, but they also appear significantly more localized than the northern magnetic polar emissions observed by Voyager 2. Lamy et al. [2012] also reported, however, that weak extended auroral arcs may have been present in some of the earlier 1998 HST images.

[12] A key difference between the magnetospheric configuration during the Voyager 2 flyby and the 2011 HST campaign, however, resulted from the $\sim 25$ year interval between them, corresponding to a little over a quarter of a Uranian year (a quarter year being 21.1 Earth years). Northern solstice conditions during the Voyager 2 flyby had thus transformed to near-autumnal equinox conditions during the HST observations (equinox occurring in December 2007). The planetary spin axis was then approximately perpendicular to the solar wind flow in the plane of the planetary orbit, with the magnetic dipole vector rotating approximately in a plane containing the solar wind velocity vector, rather than nearperpendicular to it as during the Voyager 2 flyby. It is evident in this case that the magnetospheric configuration will have been very different from that observed during the flyby as outlined in section 2.1. In the next section, we thus discuss some consequences of the equinoctial configuration, in particular concerning the formation of a magnetic tail under these circumstances, and consider their relation to the observations reported by Lamy et al. [2012].

\section{Tail Formation at Uranus During Equinoctial Conditions}

[13] Our discussion is illustrated by the sketches in Figure 1, where each diagram shows a plane containing the planet with the Sun to the left and the planet's equinoctial spin axis pointing into the plane of the diagram. The planet then rotates clockwise with increasing time. This plane is thus also approximately perpendicular to the Uranus' orbital plane, with north with respect to the Sun being directed upward relative to the planet and south with respect to the Sun being directed downward. The solar wind thus blows from left to right in each diagram, while the planet's magnetic dipole axis rotates clockwise with the planet approximately in the plane of the diagrams. The sketches illustrate conditions over one full planetary rotation, shown at quarter rotation intervals, with the instantaneous direction of the dipole being indicated by the blue arrows drawn outward from the planet in each diagram. We note that the configuration shown corresponds to autumnal equinox as pertains to the 2011 HST campaign, while at vernal equinox the spin axis would point out of rather than into the plane of the diagrams, so that the planet and magnetic dipole would then rotate anticlockwise with increasing time. With simple modification, the discussion in this section is thus also relevant to vernal equinox. The arrowed solid lines in each figure show magnetic field lines, the short-dashed lines the current sheet boundaries between open flux regimes having differing connectivity while the long-dashed lines represent the magnetopause. The effect of reconnection events as discussed below is shown by the red dotted field lines and short arrows.

[14] For definiteness, we start in Figure 1a with the magnetic dipole pointing northward with respect to the Sun (upward in the diagram) and assume for purposes of discussion that we start from a condition with no preexisting open flux in the system. In this case, the closed flux tubes located to the left of the plane defined by the spin and magnetic axes are exposed at the dayside magnetopause, such that the field there points south (with respect to the Sun). For ease of discussion, this region of closed flux is termed region " $\mathrm{A}$ " as indicated in the diagram, while that on the opposite side of the planet, to the right of the spin-dipole plane, is termed region "B." We assume that the direction of the IMF in the upstream solar wind in general fluctuates considerably on the time scale of planetary rotation. Here we then focus on an interval in which the IMF points to the north, favoring 
(a)

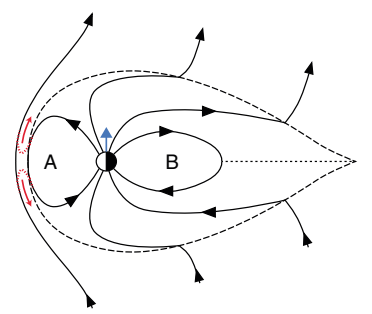

(b)

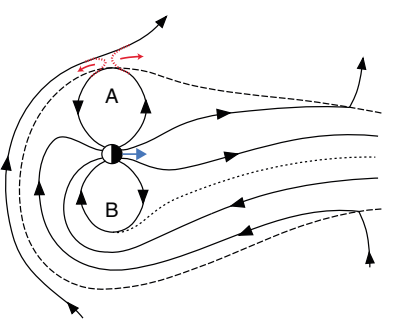

(c)

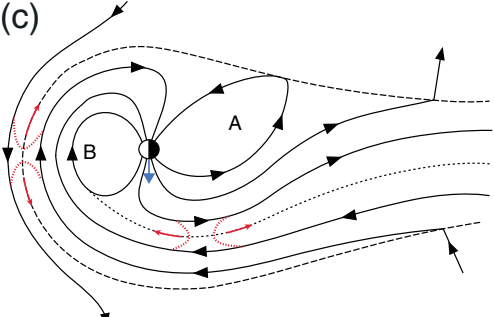

(d)

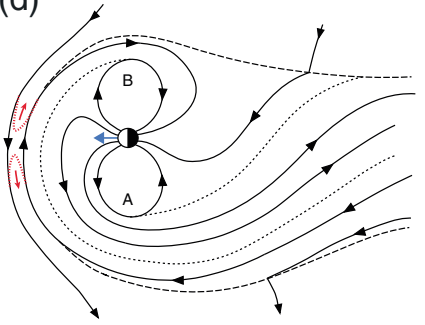

(e)

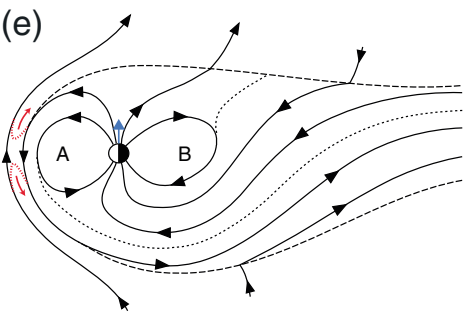

Figure 1. Sketches illustrating the evolution of open flux tubes in Uranus' magnetosphere under autumnal equinox conditions, at quarter revolution intervals over one complete $\sim 17 \mathrm{~h}$ rotation of the planet (Figures 1a-1e, respectively). Each diagram shows a plane containing the planet, with the Sun to the left and the planet's spin axis pointing into the plane. This plane is approximately perpendicular to the planet's orbital plane, with north with respect to the Sun being directed upward relative to the planet and south with respect to the Sun being directed downward. The solar wind blows from left to right in each diagram, while the planet's magnetic dipole vector, indicated by the blue arrow, rotates clockwise with the planet approximately in the plane of the diagrams. The arrowed solid lines show magnetic field lines, the short-dashed lines the boundaries between open flux regimes having differing connectivity, and the long-dashed lines the magnetopause, while the red dashed lines and short arrows indicate the effect of reconnection events in regions of high magnetic field shear. reconnection with the southward pointing closed field lines in region $\mathrm{A}$ as indicated by the red dashed lines in Figure 1a. This leads to the production of new open flux in the system and the growth of an open magnetic tail on the nightside as illustrated. Equally, we could have started the discussion with the dipole pointed to the south and the northward pointing closed field lines in region B exposed at the dayside magnetopause, forming a new open tail with a southward directed IMF, but the same overall conclusions would follow. With regard to the formation of a tail resulting from the production of open flux, we note that the time scale for open field line transport from the dayside sub-solar region into the nightside is $\sim 30 \mathrm{~min}$, corresponding to a path length along the magnetopause of $\sim 35 R_{U}$ traversed at speeds of order the solar wind speed, which is short compared with the planetary rotation period of $\sim 17 \mathrm{~h}$, such that a long tail will rapidly grow. After a quarter of a planetary rotation, for example, the open tubes will extend with the solar wind to $\sim 250 R_{U}$ downstream from the planet, more than 10 times the distance to the subsolar magnetopause, and to $\sim 500 R_{U}$ after half a rotation.

[15] The latter situations are illustrated in Figures $1 \mathrm{~b}$ and 1c, where the magnetic dipole thus points tailward in Figure $1 \mathrm{~b}$ and to the south in Figure 1c. Three effects are noted. First, as the dipole axis rotates toward the tail, the closed flux tubes in region A are decreasingly exposed at the dayside magnetopause, so that the rate of open flux production will reduce and then cease even if the IMF remains continuously northward. Second, the flux tubes in the southern lobe mapping to the south magnetic pole become draped by the planetary rotation over the closed field line region on the dayside, forming a layer of northward directed open flux adjacent to the dayside magnetopause that overlies the similarly northward directed flux in region B that now increasingly faces the Sun. This layer of open flux thus shields the closed flux inside the magnetopause from further open flux production, which would now be favored during intervals in which the IMF points southward. In the presence of an additional east-west $(Y)$ component of the IMF, this shielding may affect only part of the dayside magnetopause, rather than the complete shielding suggested by the two-dimensional sketches in the figure, but the action of the draped open field lines to reduce open flux production is clear. Third, it can be seen that as the planet rotates, the two open lobes of the "tail" will become increasingly compressed together by the solar wind flow in the region to the south of the planet, thus promoting "tail" reconnection in the current sheet between them and hence open flux closure, as indicated by the red dashed lines and arrows to the south of the planet in Figure 1c. (We note that at vernal equinox when the planet and dipole rotate anticlockwise in the plane shown, the compression instead occurs in the region to the north of the planet.) The newly closed flux tubes are added to side B of the closed field region, having initially been stripped from side A, thus requiring "return" convection in the closed field region from side B to side A. It is thus seen that each of these effects acts to prevent the growth of open flux in the system and the formation of a well-developed magnetic tail under these conditions.

[16] When "over-draped" open flux is present adjacent to the dayside magnetopause as illustrated in Figures $1 \mathrm{~b}$ and $1 \mathrm{c}$, reconnection may still occur during intervals when the 
IMF is directed southward, but then takes the form of "single lobe" reconnection with the preexisting open flux (red dashed lines and arrows on the dayside of the planet in Figure 1c). Single lobe reconnection does not change the amount of open flux in the system, but re-connects the open tubes and changes the structure of the tail on the nightside as illustrated in Figure 1d, where the dipole is now taken to point toward the Sun following the sequence in the diagrams. In this case, the lobe reconnection disconnects open flux in the southern tail from the planet, which flows tailward and eventually rejoins the solar wind, while adding the same amount of new open flux to the northern part of the tail, thus forming a more complex three-lobe tail structure. Eventually, it is possible that much of the south lobe flux will become disconnected from the planet and transferred to the north, in which case the over-draped open flux at the dayside will reverse in sign to southward pointing, such that lobe reconnection, then occurring during intervals of northward IMF, will disconnect open flux tubes connected to the north magnetic pole from the southern part of the tail and also add them to the north. This is illustrated in Figure 1e, where the dipole is taken to point to the north once more. Compression of the lobes to the south of the planet still occurs under these conditions, thus continuing to promote closure of the open flux, but now returning the newly closed flux tubes to side $\mathrm{A}$ of the closed field region, rather than to side B. In principle, lobe reconnection can continuously cycle the open flux through a three-lobe tail, with open field lines exclusively threading the northern tail magnetopause (the southern at vernal equinox) while compression of the open lobes in the southern part of the system (northern at vernal equinox) will continue to promote the closure of the open flux.

[17] Overall, these considerations thus suggest the presence of a weak and structured tail at Uranus during equinoctial conditions. Intervals of open flux production tend after some fraction of a planetary rotation (i.e., a few hours) to inhibit further production by draping over the dayside magnetopause, until that flux is either removed by "tail" reconnection and closure, or by being swept around the "sides" of the magnetosphere as noted in section 4 below. Lobe reconnection of the overdraped open flux will also contribute to tail structuring through cycling of the open flux in the system. In this situation, the region of open flux in the ionosphere near the magnetic poles will be considerably smaller than that estimated above based on Voyager 2 tail observations, such that auroral responses to solar wind-driven compressions, if present, will be correspondingly reduced in scale. In addition, precipitation from adjacent closed flux tubes resulting from "tail" reconnection events and consequent plasma injections such as those envisaged in Figure 1, similar in nature to those inferred from Voyager 2 in situ and UV data [Sittler et al., 1987; Herbert, 2009], will also be contracted around the pole, surrounding the region of reduced open flux.

\section{Discussion and Summary}

[18] The discussion in section 3 no doubt represents a highly simplified outline of what is clearly a complex magnetospheric situation at Uranus during equinox, especially when more general and varying directions of the IMF are considered. In particular, in the presence of an east-west $(Y)$ field component perpendicular to the plane of the sketches in Figure 1, the shielding of the closed flux on the dayside may not be complete as noted above, leading to the possibility of continued weak open flux production and a complex multilobe tail structure. In addition, under such circumstances, some of the open flux may also slip around the "sides" of the magnetosphere, out of the plane of the sketches in Figure 1, rather than being wound over the region of closed flux as discussed in section 3. This would produce weak open lobes on the dawn and dusk flanks of the system whose sense depends on the prevailing east-west IMF component, that are slowly twisted by planetary rotation. Reversal of the east-west component in the following IMF sector would then reverse the sense of the open flux being carried to dusk and dawn, thus again promoting reconnection between the "old" and "new" open field lines and hence destruction of the open flux.

[19] These more general scenarios are sufficiently complex that computational modeling of the large-scale system will be required to elucidate the consequences. However, the discussion in section 3 is sufficient to show that formation and maintenance of a significant open tail at Uranus under equinoctial conditions are distinctly problematical, unlike conditions near solstice (and at the other magnetized planets) when open flux can in principle be produced continuously and flow unhindered into the slowly rotating nightside tail. These arguments may thus indicate why the auroral responses to strong solar wind compressions observed by Lamy et al. [2012] were so weak and spot like, in terms of the lack of a substantial magnetic tail of open flux under the nearequinoctial conditions then prevailing. Auroras at Uranus and their responses to the solar wind are expected to be significantly more pronounced nearer to solstice, with more extended emissions bordering the expanded open flux regions, as observed by Voyager 2 (and possibly during the earlier HST observations discussed by Lamy et al. [2012]), together with significant poleward excursions resulting from open flux closure events induced by solar wind compressions, yet to be observed.

[20] Acknowledgments. This work was stimulated by observations made by the NASA/ESA Hubble Space Telescope (GO Program 12601). Laurent Lamy and Steve Milan are thanked for useful discussions and John Coxon for help with the production of the figure. The work was supported by STFC grants ST/H002480/1 and ST/K001000/1.

[21] Philippa Browning thanks Athanasios Boudouridis and another reviewer for their assistance in evaluating this paper.

\section{References}

Badman, S. V., and S. W. H. Cowley (2007), Significance of Dungey cycle flows in Jupiter's and Saturn's magnetospheres, and their identification on closed equatorial field lines, Ann. Geophys., 25, 941-951.

Badman, S. V., E. J. Bunce, J. T. Clarke, S. W. H. Cowley, J.-C. Gérard, D. Grodent, and S. E. Milan (2005), Open flux estimates in Saturn's magnetosphere during the January 2004 Cassini-HST campaign, and implications for reconnection rates, J. Geophys. Res., 110, A11216, doi:10.1029/ 2005JA011240.

Baron, R. L., T. Owen, J. Connerney, T. Satoh, and J. Harrington (1996), Solar wind control of Jupiter's auroras, Icarus, 120, 437-442.

Behannon, K. W., R. P. Lepping, E. C. Jr.Sittler, N. F. Ness, B. H. Mauk,

S. M. Krimigis, and R. L. McNutt (1987), The magnetotail of Uranus, J. Geophys. Res., 92, 15,354-15,366.

Boudouridis, A., E. Zesta, L. R. Lyons, P. C. Anderson, and D. Lummerzheim (2003), Effect of solar wind pressure pulses on the size and strength of the auroral oval, J. Geophys. Res., 108(A4), 8012, doi:10.1029/2002JA009373.

Boudouridis, A., E. Zesta, L. R. Lyons, P. C. Anderson, and D. Lummerzheim (2004), Magnetospheric reconnection driven by solar wind pressure fronts, Ann. Geophys., 22, 1367-1378.

Boudouridis, A., E. Zesta, L. R. Lyons, P. C. Anderson, and D. Lummerzheim (2005), Enhanced solar wind geoeffectiveness after a 


\section{COWLEY: URANUS' AURORAS AT EQUINOX}

sudden increase in dynamic pressure during southward IMF orientation, J. Geophys. Res., 110, A05214, doi:10.1029/2004JA010704.

Boudouridis, A., E. Zesta, L. R. Lyons, P. C. Anderson, and A. J. Ridley (2008), Temporal evolution of the transpolar potential after a sharp enhancement in solar wind dynamic pressure, Geophys. Res. Lett., 35, L02101, doi:10.1029/2007GL031766.

Broadfoot, A. L., et al. (1986), Ultraviolet spectrometer observations of Uranus, Science, 233, 74-79.

Bunce, E. J., S. W. H. Cowley, C. M. Jackman, J. T. Clarke, F. J. Crary, and M. K. Dougherty (2006), Cassini observations of the interplanetary medium upstream of Saturn and their relation to Hubble Space Telescope auroral data, Adv. Space Res., 38, 806-814.

Clarke, J. T., et al. (2005), Morphological differences between Saturn's ultraviolet aurorae and those of Earth and Jupiter, Nature, 433, 717-719.

Clarke, J. T., et al. (2009), The response of Jupiter's and Saturn's auroral activity to the solar wind, J. Geophys. Res., 114, A05210, doi:10.1029/2008JA013694.

Connerney, J. E. P., M. H. Acuña, and N. F. Ness (1987), The magnetic field at Uranus, J. Geophys. Res., 92, 15,329-15,336.

Cowley, S. W. H., S. V. Badman, E. J. Bunce, J. T. Clarke, J.-C. Gérard, D. Grodent, C. M. Jackman, S. E. Milan, and T. K. Yeoman (2005), Reconnection in a rotation-dominated magnetosphere and its relation to Saturn's auroral dynamics, J. Geophys. Res., 110, A02201, doi:10.1029/ 2004JA010796.

Desch, M. D., J. E. P. Connerney, and M. L. Kaiser (1986), The rotation period of Uranus, Nature, 322, 42-43.

Herbert, F. (2009), Aurora and magnetic field of Uranus, J. Geophys. Res., 114, A11206, doi:10.1029/2009JA014394.

Herbert, F., and B. R. Sandel (1994), Uranian aurora and its relationship to the magnetosphere, J. Geophys. Res., 99, 4143-4160.

Hubert, B., M. Palmroth, T. V. Laitinen, P. Janhunen, S. E. Milan, A. Grocott, S. W. H. Cowley, T. Pulkkinen, and J.-C. Gérard (2006), Compression of the Earth's magnetotail by interplanetary shocks directly drives transient magnetic flux closure, Geophys. Res. Lett., 33, L10105, doi:10.1029/ 2006GL026008.

Jackman, C. M., N. Achilleos, E. J. Bunce, S. W. H. Cowley, M. K. Dougherty, G. H. Jones, S. E. Milan, and E. J. Smith (2004) Interplanetary magnetic field at $\sim 9$ AU during the declining phase of the solar cycle and its implications for Saturn's magnetospheric dynamics, J. Geophys. Res., 109, A11203, doi:10.1029/2004JA010614.

Lamy, L., et al. (2012), Earth-based detection of Uranus' UV aurorae, Geophys. Res. Lett., 39, L07105, doi: 10.1029/2012GL051312.

Mauk, B. H., S. M. Krimigis, E. P. Keath, A. F. Cheng, T. P. Armstrong, L. J. Lanzerotti, G. Gloekler, and D. C. Hamilton (1987), The hot plasma and radiation environment of the Uranian magnetosphere, J. Geophys. Res., 92, 15,283-15,308.

Milan, S. E., S. W. H. Cowley, M. Lester, D. M. Wright, J. A. Slavin, M. Fillingim, C. W. Carlson, and H. J. Singer (2004), Response of the magnetotail to changes in the open flux content of the magnetosphere, J. Geophys. Res., 109, A04220, doi:10.1029/2003JA010350.

Ness, N. F., M. H. Acuña, K. W. Behannon, L. F. Burlaga, J. E. P. Connerney, R. P. Lepping, and F. M. Neubauer (1986), Magnetic fields at Uranus, Science, 233, 85-89.

Nichols, J. D., E. J. Bunce, J. T. Clarke, S. W. H. Cowley, J.-C. Gérard, D. Grodent, and W. R. Pryor (2007), Response of Jupiter's UV auroras to interplanetary conditions as observed by the Hubble Space Telescope during the Cassini fly-by campaign, J. Geophys. Res., 112, A02203, doi:10.1029/2006JA012005.

Prangé, R., et al. (2004), An interplanetary shock traced by planetary auroral storms from the Sun to Saturn, Nature, 432, 78-81.

Pryor, W. R., et al. (2005), Cassini UVIS observations of Jupiter's auroral variability, Icarus, 178, 312-326.

Sittler, E. C., Jr., K. W. Ogilvie, and R. Selesnick (1987), Survey of electrons in the Uranian magnetosphere: Voyager 2 observations, J. Geophys. Res., 92, 15,263-15,281.

Tsurutani, B. T., et al. (2001), Auroral zone dayside precipitation during magnetic storm initial phases, J. Atmos. Sol. Terr. Phys., 63, 513-522.

Zesta, E., H. J. Singer, D. Lummerzheim, C. T. Russell, L. R. Lyons, and M. J. Brittnacher (2000), The effect of the January 10, 1997, pressure pulse on the magnetosphere-ionosphere current system, in Magnetospheric Current Systems, Geophys. Monogr. Ser., vol. 118, edited by S. Ohtani, R. Fujii, M. Hesse, and R. L. Lysak, pp. 217-226, AGU, Washington, D.C.

Zhou, X., and B. T. Tsurutani (1999), Rapid intensification and propagation of the dayside aurora: Large scale interplanetary pressure pulses (fast shocks), Geophys. Res. Lett., 26, 1097-1100. 